\title{
Biodegradation kinetics of 4-fluorocinnamic acid by a consortium of Arthrobacter and Ralstonia strains
}

\author{
Syed A. Hasan · Piet Wietzes • Dick B. Janssen
}

Received: 5 March 2011/Accepted: 9 June 2011/Published online: 5 July 2011

(C) The Author(s) 2011. This article is published with open access at Springerlink.com

\begin{abstract}
Arthrobacter sp. strain G1 is able to grow on 4-fluorocinnamic acid (4-FCA) as sole carbon source. The organism converts 4-FCA into 4-fluorobenzoic acid (4-FBA) and utilizes the two-carbon side-chain for growth with some formation of 4-fluoroacetophenone as a dead-end side product. We also have isolated Ralstonia sp. strain $\mathrm{H} 1$, an organism that degrades 4-FBA. A consortium of strains G1 and H1 degraded 4-FCA with Monod kinetics during growth in batch and continuous cultures. Specific growth rates of strain G1 and specific degradation rates of 4-FCA were observed to follow substrate inhibition kinetics, which could be modeled using the kinetic models of HaldaneAndrew and Luong-Levenspiel. The mixed culture showed complete mineralization of 4-FCA with quantitative release of fluoride, both in batch and continuous cultures. Steady-state chemostat cultures that were exposed to shock loadings of substrate responded with rapid degradation and returned to steady-state in $10-15 \mathrm{~h}$, indicating that the mixed culture provided a robust system for continuous 4-FCA degradation.
\end{abstract}

S. A. Hasan · P. Wietzes · D. B. Janssen $(\square)$

Department of Biochemistry, Groningen Biomolecular

Sciences and Biotechnology Institute, University

of Groningen, Nijenborgh 4, 9747 AG Groningen,

The Netherlands

e-mail: d.b.janssen@rug.nl
Keywords Organofluorine compounds . Arthrobacter · Ralstonia $\cdot$ Biodegradation . Defluorination · 4-Fluorocinnamic acid

\section{Introduction}

Fluorinated chemicals are prominent xenobiotics that are used in pharmaceutical and agricultural applications because of their bioactivity, biological stability, high lipophilicity and ability to resist metabolic conversion (Natarajan et al. 2005; Tavener and Clark 2003). Fluoro-substitution can cause significant biological effects, such as enhanced enzyme inhibition, changes in cell-to-cell communication, disruption of transport over the membrane and inhibition of energy generation (Key et al. 1997). Industrial applications of fluoroorganics are widespread. For example, polymers of 4-fluorophenol and 4-fluorocinnamic acid (4-FCA) are used in electronic industries (Gerus et al. 2004), which can cause environmental contamination if industrial effluent is discharged untreated. The use of organofluorines in open applications and improper disposal of waste have led to their occurrence as ubiquitous environmental contaminants (Key et al. 1997).

Even though the synthesis and use of fluoroorganics is growing and they often inevitably end up in the environment, knowledge about their biodegradation is scarce. Biodegradation studies with halogenated chemicals have mostly been focused on brominated 
and chlorinated chemicals (Fetzner 1998; Hardman 1991; Janssen et al. 2001), and few papers describe the biodegradation of fluorinated compounds (Hughes et al. 2011; Murphy 2010; Murphy et al. 2011).

Treatment of 4-FCA containing waste streams from electronic industries is necessary to preserve environmental quality. Among the various techniques available for removing xenobiotic chemicals, biological treatment often is the most economical and versatile approach, as it leads to complete mineralization (Parales et al. 2002). For the design of processes for treatment of toxic waste streams, an understanding of the degradation kinetics is very important. Models that include bacterial growth and substrate utilization have been developed to describe the kinetics of contaminant biodegradation. The microbial growth rate is most often described using Monod kinetics. However, high substrate concentrations may become inhibitory to growth. Several models have been developed to quantify inhibitory effects of toxic substrates on growth and degradation. The Haldane-Andrew (Andrew 1968; Haldane 1930) and Luong-Levenspiel (Levenspiel 1980; Luong 1987) models are the most commonly used ways to describe microbial metabolism with inhibition kinetics.

Recently, we have demonstrated the possibility of partial degradation of 4-FCA by Arthrobacter sp. strain G1 and complete degradation by a mixed culture of strain G1 and Ralstonia sp. strain H1 (Hasan et al. 2011). Several strains of the genus Arthrobacter are known to utilize similar aromatic compounds which include $p$-hydroxybenzoic acid (Johnson et al. 1999), gentisic acid (Gerus et al. 2004), 4-chlorobenzoate (Marks et al. 1984; Muller et al. 1988; Zaitsev et al. 1991), mono- and dichlorinated biphenyls (Furukawa and Chakrabarty 1982), 3-aminophenol (Lechner and Straube 1988), phenol (Karigar et al. 2006), 4-fluorophenol (Ferreira et al. 2008) and a mixture of phenols (Unell et al. 2008). Members of the genus Ralstonia are also known for their potential to mineralize aromatic compounds (Baggi et al. 2004; Chen et al. 2004, 2007; Leonard and Lindly 1999; Salehi et al. 2010). The aerobic degradation of 4-FCA was reported earlier to proceed via 4-fluoro-acetophenone to form 4-fluorobenzoic acid (4-FBA) as the end product (Creaser et al. 2002; dos Santos et al. 2001; New et al. 2000). These studies were performed with activated sludge that was used for the treatment of a pharmaceutical waste. We came to an alternative degradation pathway for 4-FCA by the consortium of strain G1 and strain H1 through studying metabolites formed by whole cells and testing partially purified enzymes (Hasan et al. 2011). We found that strain G1 utilizes 4-FCA for growth by cleaving off and mineralizing the two-carbon side-chain with release of 4-FBA as the major product. Traces of 4-fluoroacetophenone were formed as a side product.

In the current study, we further evaluate the mineralization of 4-FCA by a pure and mixed cultures under batch and continuous cultivation conditions in shake flask and chemostat. We also provide the information about the degradation kinetics and the effect of shock loadings. The consortium established by the symbiotic relationship of these strains may be useful to understand and improve bioremediation of organofluorine-contaminated streams.

\section{Materials and methods}

Growth conditions

Cells of Arthrobacter sp. strain G1 and Ralstonia sp. strain $\mathrm{H} 1$ were grown aerobically at $30^{\circ} \mathrm{C}$ in flasks under rotary shaking or in a 2.51 fermentor. Growth medium (MMY) contained per liter: $5.37 \mathrm{~g}$ of $\mathrm{Na}_{2} \mathrm{H}-$ $\mathrm{PO}_{4} \cdot 12 \mathrm{H}_{2} \mathrm{O}, 1.36 \mathrm{~g}$ of $\mathrm{KH}_{2} \mathrm{PO}_{4}, 0.5 \mathrm{~g}$ of $\left(\mathrm{NH}_{4}\right)_{2} \mathrm{SO}_{4}$, $0.2 \mathrm{~g}$ of $\mathrm{MgSO}_{4} \cdot 7 \mathrm{H}_{2} \mathrm{O}$. The media were supplemented with a trace elements solution $\left(5 \mathrm{ml} \mathrm{l}^{-1}\right)$ (Hasan et al. 2011) and $10 \mathrm{mg}$ yeast extract (Difco Laboratories).

In batch culture experiments, cells of strain G1 and H1 were grown separately on 4-FCA and 4-FBA, respectively, as sole carbon source. For biodegradation experiments with cell suspensions, cells were harvested $(4,000 \times g$ for $10 \mathrm{~min})$ at mid log phase at an optical density at $450 \mathrm{~nm}$ of approximately 0.5 . Cells were washed twice with $100 \mathrm{mM}$ potassium phosphate buffer ( $\mathrm{pH}$ 6.8) and resuspended in the same buffer. To follow biodegradation of 4-FCA, cell suspensions were added to $250 \mathrm{ml}$ flasks containing $100 \mathrm{ml}$ of MMY medium supplemented with 4-FCA at a concentration ranging from 0.2 to $15 \mathrm{mM}$. To study biodegradation of 4-FBA, cells were incubated similarly in MMY medium supplemented with $2 \mathrm{mM}$ 4-FBA. Cells were incubated in a rotary shaker at $30^{\circ} \mathrm{C}$ and $150 \mathrm{rpm}$. Samples were taken with suitable time intervals, centrifuged at $16,000 \times g$ for $2 \mathrm{~min}$, and analyzed immediately by HPLC, LC-MS and ion 
chromatography. To study the kinetics of complete mineralization of 4-FCA, mixed cultures of strains $\mathrm{G} 1$ and $\mathrm{H} 1$ were inoculated into MMY medium containing 0.1-30 mM 4-FCA.

Optical densities were monitored using a spectrophotometer at a wavelength of $450 \mathrm{~nm}$. The $\mathrm{OD}_{450}$ values were then converted to dry cell mass $\left(\mathrm{OD}_{450}\right.$ of 1 corresponds to $0.16 \mathrm{~g}^{-1}$ dry weight).

\section{Growth kinetics}

For studying growth kinetics of strain G1 with 4-FCA as a limiting substrate, we used the Monod equation:

$\mu=\frac{\mu_{\max } S}{K_{s}+S}$,

where $\mu$ is the specific growth rate $\left(\mathrm{h}^{-1}\right), \mu_{\max }$ the maximum specific growth rate $\left(\mathrm{h}^{-1}\right), S$ the substrate concentration $(\mathrm{mM})$, and $K_{s}$ the half saturation substrate concentration $(\mathrm{mM})$.

For describing growth of microorganisms with inhibition at high substrate concentrations, the Haldane-Andrew model (Andrew 1968) was used, which is given by the following equation:

$\mu=\frac{\mu_{\max } S}{K_{s}+S+\frac{S^{2}}{K_{i}}}$

Where $K_{i}$ is the substrate inhibition constant (mM). Luong (1987) applied the equation of Levenspiel (1980), which is an extended Monod type model that can also be applied to describe growth inhibition at high substrate concentrations:

$\mu=\frac{\mu_{\max } S}{K_{s}+S}\left[1-\frac{S}{S_{m}}\right]^{n}$

Where $S_{m}$ is the critical inhibitor concentration above which growth stops and $n$ is an empirical constant.

In order to establish the effect of the 4-FCA concentration on growth and degradation, specific growth rates and substrate degradation rates at different 4-FCA concentrations were obtained from time course measurements of the $\mathrm{OD}_{450}$ and substrate concentration in cultures started with different 4-FCA concentrations.

Continuous culture

For growth of strains in continuous culture, we used a 31 fermentor filled with 2.51 of MMY medium. The
$\mathrm{pH}$ was maintained at 7.0 with a sterile solution of $2 \mathrm{M}$ $\mathrm{NaOH}$ added by a pump connected to a $\mathrm{pH}$ controller. The reactor was kept at $30^{\circ} \mathrm{C}$ by a temperature sensor and controller, and the agitator speed was adjusted to $250 \mathrm{rpm}$. Culture medium was supplied to the reactor vessel with a peristaltic pump. Sterile air was supplied to the fermentor by passing it through a $0.45 \mu \mathrm{m}$ pore size filter. The airflow rate was controlled with a mass flow controller. The purity of the cultures during operation of the fermentor was regularly checked by plating culture samples on NB and LB plates, which were incubated at $30^{\circ} \mathrm{C}$. When a culture attained steady state (after 5 volume changes), samples were collected for estimation of dry cell mass, fluoride release and residual 4-FCA. The values of maximum specific growth rate $\left(\mu_{\max }\right)$ were determined by the method of washout, i.e. when $D>\mu_{\max }$. The biomass decreases according to Eq. 4 (Molin 1983),

$$
\begin{aligned}
& \ln X=\left(\mu_{\max }-D\right) t+\ln X_{o} \\
& \text { or } \mu_{\max }=\frac{1}{t} \ln \frac{X}{X_{o}}+D
\end{aligned}
$$

where $X_{o}$ is the initial biomass concentration and $X$ is the biomass concentration at time $t(\mathrm{~h})$.

The Monod half-saturation constant $\left(K_{s}\right)$ was determined by analysis of substrate concentrations at different dilution rates using the Monod equation.

\section{Chemostat pulse experiments}

The effect of a pulse addition of 4-FCA on the growth of the consortium was studied by following the response after a sudden supply of $10 \mathrm{mM} 4-\mathrm{FCA}$ to a continuous culture that was operated at a dilution rate of $0.033 \mathrm{~h}^{-1}$. Steady-state conditions were disturbed by injecting 4-FCA through a septum directly into the reactor vessel to a final concentration of $10-16 \mathrm{mM}$. One sample was taken immediately after 4-FCA injection, centrifuged, and the concentrations of 4-FCA, 4-FBA and fluoride were measured. Subsequent samples were taken with suitable intervals until the entire amount of 4-FCA added was removed due to dilution and conversion by the cells. The time course of substrate concentration is given by supply to the culture, washout by dilution, and biodegradation, according to differential equation 5 : 
$\frac{\mathrm{d} S}{\mathrm{~d} t}=D\left(S_{o}-S\right)-\frac{1}{Y} \frac{\mu_{\max } S}{K_{s}+S} X$

The rate of change of biomass concentration of the bacterial consortium in continuous culture may be described by Eq. 6:

$\frac{d X}{d t}=\frac{\mu_{\max } S}{K_{s}+S} X-D X$

The rate of fluoride formation during a pulse experiment in continuous culture is described by Eq. 7:

$\frac{\mathrm{d} F}{\mathrm{~d} t}=\frac{1}{Y} \frac{\mu_{\max } S}{K_{S}+S} X-D F$

In Eqs. 5-7, $S_{o}$ is the 4-FCA concentration in the chemostat reservoir (mM), $S$ is the concentration of 4-FCA in the chemostat culture at time $t(\mathrm{mM}), F$ is the fluoride concentration in the effluent $(\mathrm{mM}), \mu_{\max }$ is the maximum specific rate of 4-FCA conversion (mmol 4-FCA transformed per gram biomass in $1 \mathrm{~h}$, $\mathrm{h}^{-1}$ ), $K_{s}$ is the Monod constant for the conversion of 4-FCA and formation of fluoride (mM), $D$ is the dilution rate $\left(\mathrm{h}^{-1}\right), X$ is the biomass concentration at time $t\left(\mathrm{~g}^{-1}\right)$, and $Y$ is the yield coefficient.

Parameter estimation for Eqs. 5, 6 and 7 was carried out using the program Scientist (MicroMath Inc., Salt Lake City, UT).

\section{Analytical methods}

Concentrations of 4-FCA, 4-FBA and their metabolites in culture supernatants were determined by reverse phase HPLC (Jasco PU-2086 pump and Jasco AS-2051 autosampler), using a Lichrosorb C18 column $(250 \times 4.6 \mathrm{~mm}, 5 \mu \mathrm{m}$ particle size $)$. The mobile phase was $0.02 \mathrm{M}$ ammonium acetate adjusted to $\mathrm{pH} 4.5$ with $70 / 30$ (v/v) acetic acid/ methanol. The injection volume was $10 \mu \mathrm{l}$, the flow rate was $0.8 \mathrm{ml} \mathrm{min}^{-1}$, and detection was at $254 \mathrm{~nm}$ with a variable UV-absorbance detector (Jasco UV2075). The reproducibility of these assays was within $5 \%$, which is better than the experiment-to-experiment variability, and therefore data that are given are representative examples of individual experiments.

LC-MS was carried out using a MicroMass ZMD equipped with a 996 Waters photodiode array detector and an Alliance 2690 separation module. HPLC conditions were as described in the previous paragraph.
The mass spectrometer scan range was from $\mathrm{m} / \mathrm{z}, 50$ to 600 and detection was in the negative ion mode. The source and desolvation temperatures were set to 125 and $150^{\circ} \mathrm{C}$, respectively. The cone and capillary voltages were set to 30 and $2.25 \mathrm{~V}$, respectively.

Fluoride measurements were performed by IC using a DX 120 ion chromatograph (Dionex, Sunnyvale, CA, USA) connected to an autosampler. This was equipped with an Alltech A-2 anion $(100 \times 4.6 \mathrm{~mm}, 7 \mu \mathrm{m})$ column and an Alltech guard $(50 \times 4 \mathrm{~mm})$ column. The injection volume was $50 \mu \mathrm{l}$. The column temperature was set to $30^{\circ} \mathrm{C}$. The eluent used was a mixture of $\mathrm{NaHCO}_{3}$ and $\mathrm{Na}_{2} \mathrm{CO}_{3}$ in deionized water at a flow rate of $1.2 \mathrm{ml} \mathrm{min}{ }^{-1}$.

\section{Results and discussion}

Kinetics of 4-FCA degradation

In order to investigate the kinetic properties of Arthrobacter sp. strain G1 and Ralstonia sp. strain $\mathrm{H} 1$, we performed a number of growth experiments in batch cultures. In MMY medium, strain G1 grew exponentially between 3 and $16 \mathrm{~h}$ with specific growth rate of $0.22 \mathrm{~h}^{-1}$ (Fig. 1) when $2 \mathrm{mM}$ 4-FCA was supplied as the sole source of carbon and energy. Both 4-FBA and 4-fluoroacetophenone (small amounts) were observed as products by LS-MS. Strain H1 degraded 4-FBA, forming 4-fluorocatechol (4-FC) which led to ring fission with fluoride release (Fig. 2). Traces of 4-FC remained in the culture medium for a prolonged period.

In order to examine the toxic effects of 4-FCA and its degradation products on growth, cells of strain G1 were incubated in MMY medium containing increasing concentrations $(0.2-15 \mathrm{mM})$ of 4-FCA. For comparison, mixed cultures of strains $\mathrm{G} 1$ and $\mathrm{H} 1$ were incubated in separate flasks containing MMY medium supplemented with $0.4-30 \mathrm{mM}$ 4-FCA. For each batch culture with a certain 4-FCA concentration, cell growth was measured at a function of time. The specific growth rates at different 4-FCA concentrations were calculated by measuring the slope of increasing $\mathrm{OD}_{450}$ and average cell density of every data set obtained at different 4-FCA concentrations.

From the results, it is quite clear that the specific growth rate increases with an increase in substrate concentration until a maximum value is reached. 


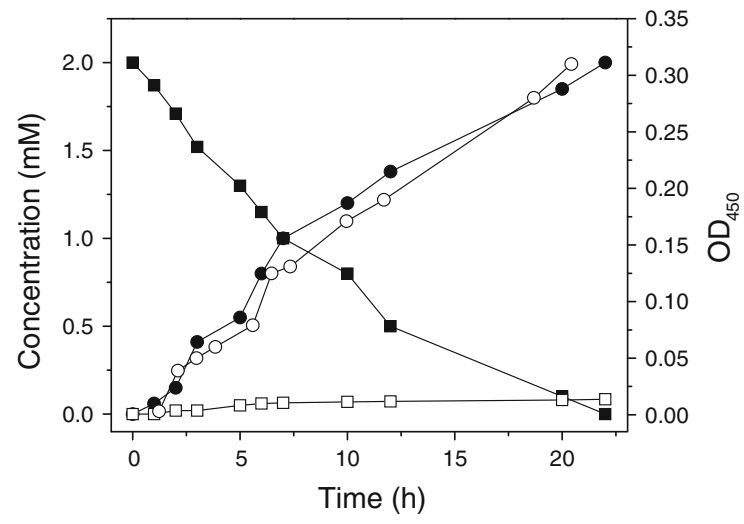

Fig. 1 Growth of strain G1 in MMY medium supplemented with $2 \mathrm{mM}$ 4-FCA. Symbols: filled square 4-FCA concentration; filled circle 4-FBA concentration; open circle optical density at $450 \mathrm{~nm}$; and open square 4-fluoroacetophenone concentration

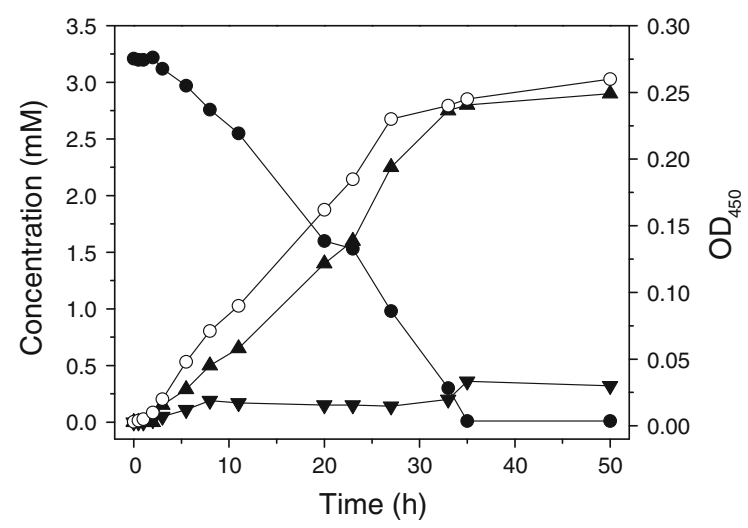

Fig. 2 Growth of Ralstonia sp. H1 in MMY supplemented with $3.25 \mathrm{mM}$ 4-FBA. Symbols: filled circle 4-FBA concentration; open circle optical density at $450 \mathrm{~nm}$; filled triangle $\mathrm{F}^{-}$ concentration; inverted triangle 4-FC concentration

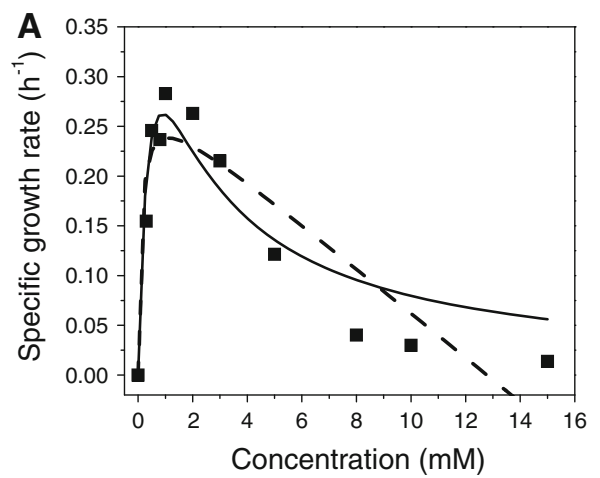

Fig. 3 Specific growth rates of (A) Arthrobacter sp. G1 and (B) a mixed culture of strain G1 and Ralstonia sp. H1 at different 4-FCA concentrations and fitting with the kinetic
However, above $2 \mathrm{mM}$ in pure culture (Fig. 3A) and above $4 \mathrm{mM}$ in case of the mixed culture (Fig. 3B), the specific growth rate started to decrease, indicating substrate inhibition of growth. This may be due to cell damage or disruption of membrane integrity at higher 4-FCA concentrations (Sikkema et al. 1995; Weber and de Bont 1996).

The Monod model was used to determine the growth parameters and the Haldane-Andrew and Luong-Levenspiel models were used to quantify the effects of substrate inhibition on growth rates. To fit the data and estimate the values of the biokinetic constants of these models, non-linear regression was done with Microcal Origin 7. The parameters estimated by using these models are summarized in Table 1 . The Haldane-Andrew model give $K_{i}$ values that indicate the sensitivity of the culture to substrate inhibition above a concentration where which the specific growth rate declines. The higher $K_{i}$ value of the mixed culture $(4.1 \mathrm{mM})$ compared to the $K_{i}$ of the pure culture $(2.5 \mathrm{mM})$ indicated a higher tolerance to substrate of the mixed culture. This higher resistance to increased substrate levels of the mixed culture was not accompanied by a higher growth rate. Maybe the sequential nature of the degradation process or the need to accumulate 4-FBA before growth of strain $\mathrm{H} 1$ can start is responsible for this lower apparent maximum growth rate. Alternatively, strain H1 may form transient metabolites that reduce the 4-FCA consumption rate.

The Luong-Levenspiel model uses a critical substrate concentration $\left(S_{m}\right)$, at which the growth rate falls to zero. A high $S_{m}(24 \mathrm{mM})$ was found for the mixed culture, indicating that it can survive and

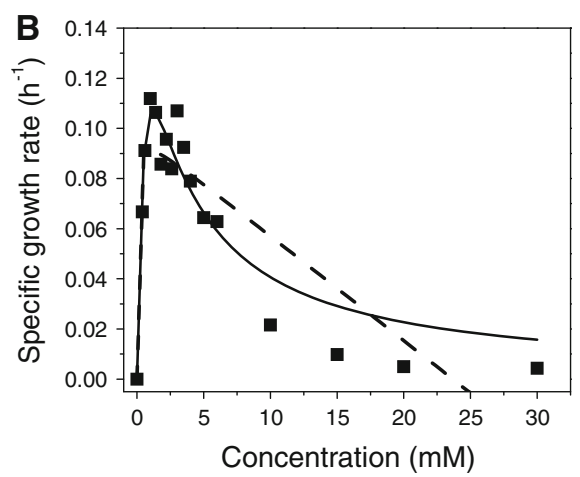

models. Symbols: filled square experimental data for [4-FCA]; solid line Haldane-Andrew regression curve; and dashed line Luong-Levenspiel regression curve 
Table 1 Kinetic parameters with Monod, Haldane-Andrew, and Luong-Levenspiel models for batch conditions with (a) a pure culture of Arthrobacter sp. strain G1 and (b) a mixed culture of strains G1 and Ralstonia sp. strain H1, growing in MMY supplemented with 4-FCA

\begin{tabular}{|c|c|c|c|c|c|c|}
\hline \multirow{2}{*}{$\begin{array}{l}\text { Kinetic } \\
\text { parameter }\end{array}$} & \multicolumn{3}{|c|}{ (a) 4-FCA growth kinetics in pure culture } & \multicolumn{3}{|c|}{ (b) 4-FCA growth kinetics in mixed culture } \\
\hline & $\begin{array}{l}\text { Monod } \\
\text { model }\end{array}$ & $\begin{array}{l}\text { Haldane-Andrew } \\
\text { model }\end{array}$ & $\begin{array}{l}\text { Luong-Levenspiel } \\
\text { model }\end{array}$ & $\begin{array}{l}\text { Monod } \\
\text { model }\end{array}$ & $\begin{array}{l}\text { Haldane-Andrew } \\
\text { model }\end{array}$ & $\begin{array}{l}\text { Luong-Levenspiel } \\
\text { model }\end{array}$ \\
\hline$\mu_{\max }\left(\mathrm{h}^{-1}\right)$ & 0.37 & 0.42 & 0.40 & 0.10 & 0.16 & 0.15 \\
\hline $\begin{array}{l}K_{s}(\mathrm{mM}), K_{m} \\
\quad(\mathrm{mM})\end{array}$ & 0.11 & 0.52 & 0.14 & 0.11 & 0.60 & 0.30 \\
\hline$K_{i}(\mathrm{mM})$ & - & 2.50 & - & - & 4.10 & - \\
\hline$S_{m}(\mathrm{mM}), n$ & - & - & $13.1, n=1$ & - & - & $24.8, n=1$ \\
\hline
\end{tabular}

grow at quite high levels of 4-FCA. In case of the pure culture of strain G1, a two-fold lower $S_{m}$ for 4-FCA was found. Of the inhibition models, the Haldane-Andrew model gave a somewhat better fit to our data than the Luong-Levenspiel model.

Kinetics of 4-FCA degradation and growth of strain G1 in chemostat culture

In order to evaluate the kinetics of strain G1 in continuous culture, we measured growth and 4-FCA removal in a chemostat bioreactor that was inoculated to an $\mathrm{OD}_{450}$ of 0.013 with a batch culture of strain $\mathrm{G} 1$ pregrown on $5 \mathrm{mM}$ 4-FCA. Growth of strain G1 with $5 \mathrm{mM}$ 4-FCA was followed in batch mode to an $\mathrm{OD}_{450}$ of 0.81 . As soon as the stationary growth phase was reached, the culture was switched to continuous mode. Growth kinetics were subsequently studied at various dilution rates, starting with $0.007 \mathrm{~h}^{-1}$ and increasing until the critical dilution rate was reached (Fig. 4A).

The $\mu_{\max }$ of strain G1 on 4-FCA determined under washout conditions (using Eq. 4) was $0.042 \mathrm{~h}^{-1}$.
Steady states were established at dilution rates between 0.007 and $0.038 \mathrm{~h}^{-1}$. The growth of strain G1 followed Monod kinetics. The $K_{s}$ was $41 \mu \mathrm{M}$ as calculated using the Monod equation. Cell mass was almost constant up to a dilution rate of $0.038 \mathrm{~h}^{-1}$, but at higher dilution rates it decreased sharply. The growth yield was almost constant during steady states and it was determined as $18.8 \mathrm{~g}$ of cell dry-mass per mole of substrate. No wall growth of cells was observed and no formation of fluoride occurred during the course of the experiment, but the concentration of 4-FBA was $4.8 \pm 0.1 \mathrm{mM}$ up to the maximum dilution rate of $0.06 \mathrm{~h}^{-1}$. The concentration of 4-FBA in the fermentor decreased sharply beyond $0.06 \mathrm{~h}^{-1}$ and was reduced to almost zero at a higher dilution rate.

To study the complete biodegradation of 4-FCA, a consortium of strains G1 and H1 was inoculated in a chemostat with a continuous supply of $10 \mathrm{mM} 4$-FCA. Cell mass and concentrations of 4-FCA, 4-FBA and $\mathrm{F}^{-}$ were measured at different dilutions rates. The culture density was almost constant up to a dilution rate of $0.15 \mathrm{~h}^{-1}$, but decreased sharply with higher dilution

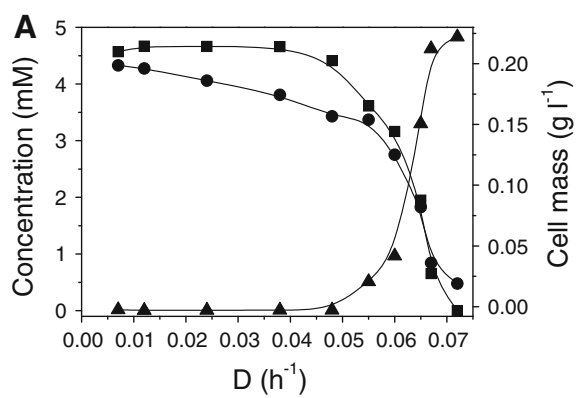

Fig. 4 Utilization of 4-FCA in continuous culture at different dilution rates. A $5 \mathrm{mM}$ 4-FCA with a pure culture of Arthrobacter sp. G1 and B $10 \mathrm{mM}$ 4-FCA with a consortium

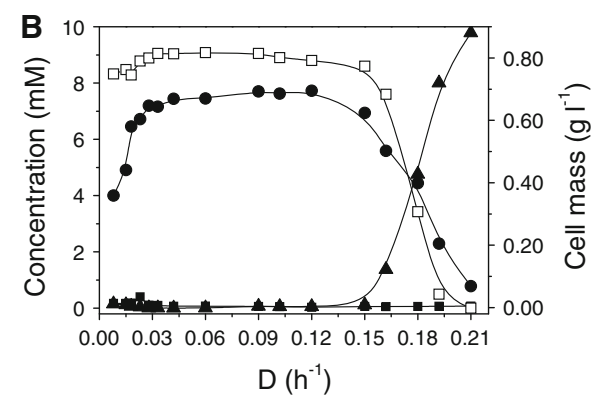

of Arthrobacter sp. G1 and Ralstonia sp. H1. Symbols: filled square 4-FBA concentration; filled circle biomass; filled triangle 4-FCA concentration; open square $\mathrm{F}^{-}$concentration 
rates (Fig. 4B). The residual concentration of 4-FCA was below $0.05 \mathrm{mM}$ from 0.008 to $0.15 \mathrm{~h}^{-1}$, and increased sharply at higher dilution rates. The apparent $K_{s}$ value of the consortium was $47 \mu \mathrm{M}$ and the $\mu_{\max }$ obtained was $0.11 \mathrm{~h}^{-1}$, which is higher than what is obtained in batch culture or with a pure culture of strain G1 in the chemostat.

Irrespective of the main product of 4-FCA degradation, the low values of $K_{s}$ indicate that under both pure and mixed culture conditions the bacterial cultures are capable of efficient degradation of 4-FCA. A low cell yield was noted during operation of the reactor when a pure culture of strain G1 was employed to degrade 4-FCA at dilution rates $0.007-0.038 \mathrm{~h}^{-1}$. At a two-fold higher substrate concentration, the cell yield was four-fold higher for the consortium of strains $\mathrm{G} 1$ and $\mathrm{H} 1$ between dilution rates of $0.033-0.12 \mathrm{~h}^{-1}$ (Fig. 4), which is in agreement with strain $\mathrm{H} 1$ being responsible for mineralization of 4-FBA, the end product of 4-FCA side-chain degradation by strain G1.

The $\mu_{\max }$ values determined for strain G1 (using Eq. 4, Fig. 4A) and for the consortium of strains G1 and H1 (Fig. 4B) were lower than the $\mu_{\max }$ values determined under wash-out conditions. In contrast, an early wash-out at $D<\mu_{\max }$ was reported for a multispecies culture growing aerobically on 6-aminonaphthalene-2-sulphonic acid (Diekmann et al. 1988) and another one growing anaerobically on phenol (Khoury et al. 1992). Although there is no clear explanation for the latter, a possible cause could

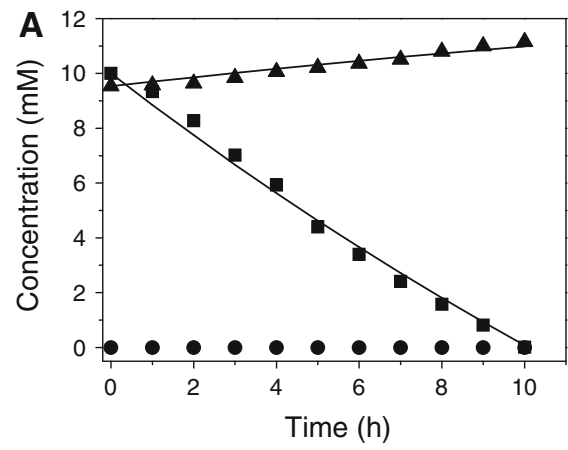

Fig. 5 Effect of 4-FCA shock loadings on substrate depletion and fluoride formation by a consortium of Arthrobacter sp. G1 and Ralstonia sp. $\mathrm{H} 1$ growing in a bioreactor that is continuously supplied with $10 \mathrm{mM}$ 4-FCA at a rate of $0.033 \mathrm{~h}^{-1}$. A A pulse of $10 \mathrm{mM} 4$-FCA was injected directly into the chemostat vessel. 4-FCA (filled square) was depleted (maximal rate $2.8 \mathrm{mmol} \mathrm{h}^{-1} \mathrm{~g}^{-1}$ cells) and fluoride (filled triangle) was released $\left(1.23 \mathrm{mmol} \mathrm{h}^{-1} \mathrm{~g}^{-1}\right)$. B A pulse of be early wash-out of a stabilizing escorting species which is not directly involved in the degradation process. In our system, both strains of the mixed culture are involved in the degradation of substrate and metabolites. The possibility to increase the dilution rate to values exceeding the $\mu_{\max }$ determined by the wash-out experiment may be due to adaptation of the cells to higher flow rates if they are kept in the chemostat in the chemostat for a prolonged period.

Effect of shock loadings of 4-FCA

to the chemostat culture

The capability of the consortium of strains G1 and H1 to degrade shock loadings of 4-FCA in continuous mode was tested by pulse additions of 10 and $16 \mathrm{mM}$ of 4-FCA to a steady-state culture growing on $10 \mathrm{mM}$ 4-FCA at a dilution rate of $0.033 \mathrm{~h}^{-1}$ (Fig. 5). Before the pulse, the steady-state concentration of 4-FCA was below the detection limit $(<0.02 \mathrm{mM})$ and the fluoride concentration was $9.5 \mathrm{mM}$. After a pulse, the consortium completely removed the extra added 4-FCA in 10 or $15 \mathrm{~h}$, respectively. During consumption of pulsed 4-FCA, cell growth resumed and the same steady-state conditions as before the pulse addition of 4-FCA were obtained.

A model based on Monod kinetics (Eqs. 5-7) was appropriate for describing the conversion of 4-FCA during the pulse experiments (Fig. 5), because no inhibition of growth or degradation was observed. Only 53 and $60 \%$ of the expected liberated fluorine

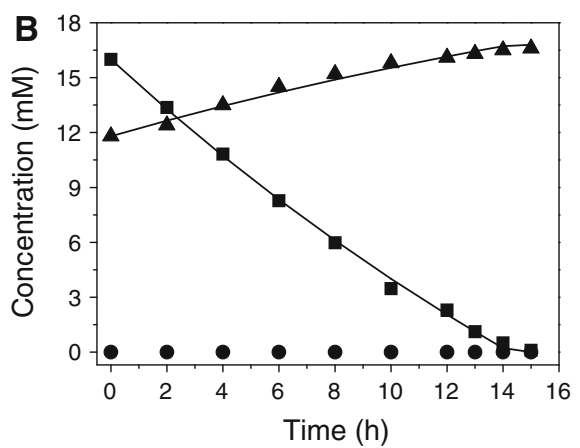

$16 \mathrm{mM}$, followed by depletion of 4-FCA (filled square) at a maximal rate of $3.0 \mathrm{mmol} \mathrm{h}^{-1} \mathrm{~g}^{-1}$ cells and fluoride (filled triangle) formation occurred at $1.22 \mathrm{mmol} \mathrm{h}^{-1} \mathrm{~g}^{-1}$ cells. The residual concentration of 4-FBA (filled circle) in the reactor remained below $0.01 \mathrm{mM}$. The solid lines show the fit with the Monod equation (5 and 7) for the removal of 4-FCA and release of fluoride 
was detected as free fluoride after the 10 and $16 \mathrm{mM}$ pulses of 4-FCA, respectively. This indicates formation of some intermediate organofluorines that were washed out and remained undetected in the effluent. Earlier, we have shown that 4-fluoroacetophenone may be formed as a side product, and transient accumulation of 4-FBA, 4-FC, and other products may occur (Hasan et al. 2011).

The maximum 4-FCA conversion rates $\left(\mu_{\max }\right)$ in the case of 10 and $16 \mathrm{mM}$ pulses of 4-FCA were estimated as 2.8 and $3.0 \mathrm{mmol} \mathrm{h}^{-1} \mathrm{~g}^{-1}$ cell dry weight, respectively. The maximum fluoride formation rate for 10 and $16 \mathrm{mM}$ of 4-FCA could be estimated to 1.23 and $1.22 \mathrm{mmol} \mathrm{h}^{-1} \mathrm{~g}^{-1}$ cells, respectively.

The removal of a high 4-FCA concentration and the final return to the initial conditions indicated that the consortium of strains G1 and H1 is highly resistant to shock loadings and can withstand a temporary exposure to a high concentration of 4-FCA in continuous culture. The increasing biomass concentration and the quick depletion of 4-FCA from the bioreactor indicates that the metabolites formed by side-chain cleavage of 4-FCA, especially 4-FBA, are not toxic for strain $\mathrm{H} 1$, and the consortium readily consumes these metabolites.

\section{Conclusion}

The data reported here show that Arthrobacter sp. strain G1 which can degrade 4-FCA by removing the side-chain of two-carbon atoms, can form a consortium with Ralstonia sp. strain H1. The mixed culture has a high tolerance to 4-FCA toxicity both in batch mode and continuous culture. It can also absorb shock loadings of higher concentrations of 4-FCA. This feature potentially enables the consortium to be used for the treatment of industrial wastewater or bioaugmentation of contaminated soils.

Acknowledgments We thank Theodora Tiemersma for help in LC-MS analysis. S. A. Hasan gratefully acknowledges the Higher Education Commission (HEC), Government of Pakistan, for financial support.

Open Access This article is distributed under the terms of the Creative Commons Attribution Noncommercial License which permits any noncommercial use, distribution, and reproduction in any medium, provided the original author(s) and source are credited.

\section{References}

Andrew JF (1968) A mathematical model for continuous culture of microorganisms utilizing inhibitory substrates. Biotechnol Bioeng 10:707-723

Baggi G, Cavalca L, Francia P, Zangrossi M (2004) Chlorophenol removal from soil suspensions: effects of a specialized microbial inoculum and a degradable analogue. Biodegradation 15:153-160

Chen W-M, Chang J-S, Wu C-H, Chang S-C (2004) Characterization of phenol and trichloroethene degradation by the rhizobium Ralstonia taiwanensis. Res Microbiol 155: 672-680

Chen B-Y, Chen W-M, Chang J-H (2007) Optimal biostimulation strategy for phenol degradation with indigenous rhizobium Ralstonia taiwanensis. J Hazard Mater B1 39:232-237

Creaser C, dos Santos LF, Lamarca DG, New A, Wolff JC (2002) Biodegradation studies of 4-fluorobenzoic acid and 4-fluorocinnamic acid: an evaluation of membrane inlet mass spectrometry as an alternative to high performance liquid chromatography and ion chromatography. Anal Chim Acta 454:137-145

Diekmann RB, Nortemann B, Hempel DC, Knackmuss H-J (1988) Degradation of 6-aminonaphthalene-2-sulfonic acid by mixed cultures: kinetic analysis. Appl Microbiol Biotechnol 29:85-88

dos Santos LMF, Spicq A, New AP, Biundo GL, Wolff JC, Edwards A (2001) Aerobic biotransformation of 4-fluorocinnamic acid to 4-fluorobenzoic acid. Biodegradation 12:23-29

Ferreira MIM, Marchesi JR, Janssen DB (2008) Degradation of 4-fluorophenol by Arthrobacter sp. strain IF1. Appl Microbiol Biotechnol 78:709-717

Fetzner S (1998) Bacterial dehalogenation. Appl Microbiol Biotechnol 50:633-657

Furukawa K, Chakrabarty AM (1982) Involvement of plasmids in total degradation of chlorinated biphenyls. Appl Environ Microbiol 44:619-626

Gerus I, Glushchenko A, Kurioz Y, Reznikov Y, Tereshchenko O (2004) Sticking of liquid crystal on photosensitive polymer layers. Opto-Electron Rev 12:281-284

Haldane JSB (1930) Enzymes, longmans, green, UK. Republished by MIT Press, Cambridge, MA (1965)

Hardman DJ (1991) Biotransformation of halogenated compounds. Crit Rev Biotechnol 11:1-40

Hasan SA, Ferreira MIM, Koetsier MJ, Arif MI, Janssen DB (2011) Complete biodegradation of 4-fluorocinnamic acid by a consortium comprising Arthrobacter sp. strain G1 and Ralstonia sp. strain H1. Appl Environ Microbiol 77: 572-579

Hughes D, Clark BR, Murphy CD (2011) Biodegradation of polyfluorinated biphenyl in bacteria. Biodegradation 22:741-749

Janssen DB, Oppentocht JE, Poelarends GJ (2001) Microbial dehalogenation. Curr Opin Biotechnol 12:254-258

Johnson TA, Sims GK, Ellsworth TR, Balance AR (1999) Effects of moisture and sorption on bioavailability of $p$ hydroxybenzoic acid to Arthrobacter sp. in soil. Microbiol Res 153:349-353 
Karigar C, Mahesh A, Nagenahalli M, Yun DJ (2006) Phenol degradation by immobilized cells of Arthrobacter citreus. Biodegradation 17:47-55

Key BD, Howell RD, Criddle CS (1997) Fluorinated organics in the biosphere. Environ Sci Technol 31:2445-2454

Khoury N, Wolfgang D, Kampfer P (1992) Anaerobic degradation of phenol in batch and continuous cultures by a denitrifying bacterial consortium. Appl Microbiol Biotechnol 37:524-528

Lechner U, Straube G (1988) Degradation of 3-aminophenol by Arthrobacter sp. mA3. J Basic Microbiol 28:629-637

Leonard D, Lindly NA (1999) Growth of Ralstonia eutropha on inhibitory concentration of phenol: diminished growth can be attributed to hydrophilic perturbation of phenol hydroxylase activity. Enzym Microb Technol 25:271-277

Levenspiel O (1980) The Monod equation: a revisit and a generalization to product inhibition situations. Biotechnol Bioeng 22:1671-1687

Luong JHT (1987) Generalization of Monod kinetics for analysis of growth data with substrate inhibition. Biotechnol Bioeng 29:242-248

Marks TS, Wait R, Smith AR (1984) The origin of the oxygen incorporated during the dehalogenation/hydroxylation of 4-chlorobenzoate by an Arthrobacter sp. Biochem Biophys Res Commun 124:669-674

Molin G (1983) Measurement of the maximum specific growth-rate in chemostat of Pseudomonas sp. with different abilities for biofilm formation. Eur J Appl Microbiol Biotechnol 18:303-307

Muller R, Oltmanns RH, Lingens F (1988) Enzymatic dehalogenation of 4-chlorobenzoate by extracts from Arthrobacter sp. SU DSM 20407. Biol Chem HoppeSeyler 369:567-571

Murphy CD (2010) Biodegradation and biotransformation of organofluorine compounds. Biotechnol Lett 32:351-359
Murphy CD, Clark BR, Amadio J (2009) Metabolism of fluoroorganic compounds in microorganisms: impacts for the environment and the production of fine chemicals. Appl Microbiol Biotechnol 84:617-629

Natarajan R, Azerad R, Badet B, Copin E (2005) Microbial cleavage of C-F bond. J Fluor Chem 126:425-436

New AP, dos Santos LMF, Biundo GL, Spicq A (2000) Analytical techniques used for monitoring the biodegradation of fluorinated compounds in waste streams from pharmaceutical production. J Chromatogr A 889:177-184

Parales RE, Bruce NC, Schmid A, Wackett LP (2002) Biodegradation, biotransformation and biocatalysis (B3). Appl Environ Microbiol 68:4699-4709

Salehi Z, Sohrabi M, Vahabzadeh F, Fatemi S, Kawase Y (2010) Modeling of $p$-nitrophenol biodegradation by Ralstonia eutropha via application of the substrate inhibition concept. J Hazard Mater 177:582-585

Sikkema J, de Bont FA, Poolman B (1995) Mechanisms of membrane toxicity of hydrocarbons. Microbiol Rev 59:201-222

Tavener SJ, Clark FH (2003) Can fluorine chemistry be green chemistry? J Fluor Chem 123:31-36

Unell M, Nordin K, Jernberg C, Stenstrom J, Jansson JK (2008) Degradation of mixtures of phenolic compounds by Arthrobacter chlorophenolicus A6. Biodegradation 19:495-505

Weber FJ, de Bont JA (1996) Adaptation mechanisms of microorganisms to the toxic effects of organic solvents on membranes. Biochim Biophys Acta 1286:225-245

Zaitsev GM, Tsoi TV, Grishenkov VG, Plotnikova EG, Boronin AM (1991) Genetic control of degradation of chlorinated benzoic acids in Arthrobacter globiformis, Corynebacterium sepedonicum and Pseudomonas cepacia strains. FEMS Microbiol Lett 65:171-176 